\title{
How to Understand PM2.5
}

\author{
Bowen Wei \\ School of North China Electric Power University, Baoding 071000, China \\ wenuf@sina.com
}

Keyword: PM2.5, multiple linear regression, principal component analysis

\begin{abstract}
In view of the factors that affect PM2.5, we set up two models. Multiple linear regression models were used in the establishment of internal factors. Consider that there are too many external factors, we adopt the dimension reduction method, using the principal component analysis method. Through the internal analysis, we found that $\mathrm{CO}$ is the main factor that affects the value of PM2.5. Through the external analysis, we found that rainfall is the main factor affecting the value of PM2.5.

When it comes to the relationship between PM2.5 and the haze, we build model three. We learn that AQI is a major index reflecting the weather conditions, so we transform the study of relationship between PM2.5 and the haze into a study of the relationship between PM2.5 and AQI. After a scatter plot analysis, we use a linear regression model. After residual analysis, we find that the fitting degree of the model is very high. From this, we come to the conclusion that PM2.5 caused by the haze, while the haze reflects the high value of PM2.5

Our models fit the reality well, which can help people understand PM2.5 more scientifically and intuitively. These analyses are also helpful in the management of air pollution. In the future, we can further refine the model from the angle of time and space.
\end{abstract}

\section{Introduction}

Haze is the general expression about excessive levels of suspended particulate matter content in atmosphere. With the deterioration of air quality, fog and haze phenomenon increased. In many areas of China, the haze weather phenomenon is incorporated into the fog as a disaster weather warning. The haze has gained wide attention since it appeared in 2013.According to data published by the Ministry of environmental protection in China, AQI of Baoding often exceeds 400 in a few days in a row, that is, "serious pollution", which caused a serious impact on local people's daily lives.

In the study of the main factors affecting the haze, main factors was analyzed affecting on the national large-scale haze phenomenon in November 2013 by using two kinds of statistical analysis methods: non parametric statistical binding method of multivariate regression factor analysis and correspondence analysis method in multivariate statistical analysis. Based on results of the above two kinds of methods and combined with the western developed countries' governance experience on haze pollution, effective methods were proposed to alleviate the haze in China [1].

In the study of the relationship between PM2.5 and haze, a research team used PM2.5/AQI ratio to measure the possibility scale of PM2.5 in haze weather. This ratio can directly characterize PM2.5 in air (haze) influence, arriving at the conclusion that PM2.5 is closely related to haze weather [2].

\section{Assumptions and Notations}

To facilitate the construction of the model, we make the following assumptions:

- The data in the Baoding Environmental Protection Bureau and other authoritative website is accurate.

- There is no strict divided boundaries between PM2.5/AQI. That is to say, the values of 
PM2.5/AQI is at or near the limit, even can be thought in any interval.

- Sunny and thunderstorms weather will not appear fog and haze.

- The error of the estimated weather condition from the picture can be ignored.

We use a list of symbols (cf. Table 1) for simplification of expression.

Table 1. Notations (in the order of model)

\begin{tabular}{ccc}
\hline Symbol & Definition & Notes \\
\hline \hline AQI & Model One & \\
$y$ & air quality index & Table 2 \\
$x$ & dependent variable & Equation (1) \\
$\beta$ & independent variable & Equation (1) \\
$\varepsilon$ & partial regression coefficient & Equation (1) \\
CV & residual & Equation (1) \\
CR & Model Two & Table 5 \\
CCR & contribution rate & Table 5 \\
& cumulative contribution rate & \\
& Model Three & \\
& The same as Model One & \\
\end{tabular}

\section{The model}

Under certain conditions, $\mathrm{SO}_{2}, \mathrm{NO}, \mathrm{NO}_{2}$ in AQI detection indicators is the main gaseous objects before PM2.5 formed [3].

\subsection{Model One}

We build model one to analyze the internal factors affecting PM2.5. Model one is called multiple linear regression model.

We collect data from some professional web [4]. The detection data range from 2015 January 1 to 2015 December 31 in Baoding. We use Sampling method and monthly selected No. 5, 15 and 25 as samples, monitoring the primary pollutants for $\mathrm{PM} 10, \mathrm{SO}_{2}, \mathrm{CO}, \mathrm{NO}_{2}, \mathrm{O}_{3}$. The initial data is as follows:

Table 2. The Initial Data of Five Main Factors

\begin{tabular}{cccccccc}
\hline Date & $\mathrm{AQI}$ & $\mathrm{PM} .5$ & $\mathrm{PM} 10$ & $\mathrm{SO}_{2}$ & $\mathrm{CO}$ & $\mathrm{NO}_{2}$ & $\mathrm{O}_{3}$ \\
\hline \hline 1.05 & 327 & 265.7 & 450 & 149.9 & 5.512 & 113.3 & 49 \\
1.15 & 365 & 299.7 & 462.8 & 97.5 & 4.861 & 82.7 & 13 \\
1.25 & 163 & 126.3 & 181.6 & 75.5 & 2.603 & 47 & 28 \\
2.05 & 112 & 79.5 & 137.3 & 115.1 & 2.162 & 47.7 & 82 \\
2.15 & 264 & 206.8 & 364.9 & 125.9 & 3.967 & 70.6 & 51 \\
2.25 & 184 & 139.2 & 253.5 & 64.4 & 2.213 & 36 & 82 \\
3.05 & 141 & 106.6 & 175 & 108.5 & 1.596 & 57.4 & 85 \\
3.15 & 198 & 132.3 & 289.3 & 99.2 & 2.166 & 70.6 & 110 \\
3.25 & 174 & 128.3 & 243.8 & 50.4 & 2.166 & 70.5 & 114 \\
4.05 & 112 & 69.2 & 130.1 & 17.1 & 1.017 & 34 & 108 \\
4.15 & 194 & 114.4 & 252.8 & 63.7 & 1.517 & 57.8 & 125 \\
4.25 & 126 & 90.9 & 175 & 52.1 & 1.254 & 46.9 & 233 \\
5.05 & 116 & 84.2 & 151.7 & 47.8 & 1.092 & 49.7 & 190 \\
5.15 & 72 & 39.6 & 85.6 & 20.7 & 0.567 & 34.3 & 159 \\
5.25 & 159 & 105.3 & 162.8 & 56.7 & 1.164 & 40.9 & 281 \\
6.05 & 90 & 51.9 & 127.3 & 42.6 & 0.729 & 45.5 & 216 \\
6.15 & 121 & 84.4 & 165.4 & 47.3 & 1.267 & 59.5 & 266
\end{tabular}




\begin{tabular}{cccccccc}
6.25 & 97 & 71 & 113.3 & 22.6 & 0.81 & 29.8 & 194 \\
7.05 & 95 & 56.1 & 90 & 19.9 & 0.796 & 30.5 & 225 \\
7.15 & 105 & 76.5 & 134.7 & 26.9 & 0.927 & 51 & 146 \\
7.25 & 121 & 91.5 & 155.3 & 24.2 & 1.097 & 35.9 & 238 \\
8.05 & 132 & 98.8 & 169.8 & 18.7 & 1.426 & 30.5 & 172 \\
8.15 & 116 & 80 & 140.2 & 17.6 & 0.683 & 48.9 & 165 \\
8.25 & 49 & 23.3 & 53.5 & 10.1 & 0.485 & 26.5 & 126 \\
9.05 & 51 & 33.6 & 52.6 & 9.7 & 0.501 & 17.1 & 102 \\
9.15 & 128 & 96.9 & 168 & 18.8 & 0.99 & 64.2 & 178 \\
9.25 & 60 & 32 & 71.6 & 17.7 & 0.503 & 38 & 122 \\
10.05 & 228 & 179.7 & 280.2 & 32.6 & 1.781 & 97.8 & 217 \\
10.15 & 231 & 167.9 & 287 & 40.9 & 2.32 & 89.6 & 266 \\
10.25 & 59 & 26.3 & 68.9 & 47 & 0.872 & 67.3 & 35 \\
11.05 & 149 & 100.8 & 176.6 & 34.2 & 1.084 & 73.1 & 46 \\
11.15 & 202 & 153.8 & 225.5 & 59.5 & 3.793 & 98.9 & 33 \\
11.25 & 79 & 57.3 & 70.4 & 58.1 & 1.676 & 41.8 & 60 \\
12.05 & 284 & 237.8 & 323.3 & 118.2 & 5.189 & 99.8 & 11 \\
12.15 & 48 & 21.5 & 51.3 & 28.4 & 0.737 & 23.5 & 69 \\
12.25 & 307 & 250.8 & 375.8 & 61.6 & 6.698 & 110.2 & 23 \\
\hline
\end{tabular}

Then we established a multivariate linear regression model based on PM2.5 as the dependent variable and SO2 CO NO2 O3 PM10 as the independent variable.

$$
y=\beta_{0}+\beta_{1} x_{1}+\beta_{2} x_{2}+\beta_{3} x_{3}+\beta_{4} x_{4}+\beta_{5} x_{5}+\varepsilon
$$

The parameters of the $\beta$, from $\beta_{0}$ to $\beta_{5}$ are regression coefficients remained to be estimated, $\varepsilon$ is random error (roughly follows a mean zero normal distribution) .

Table 3. Results of Linear Regression Model

\begin{tabular}{ccllll}
\hline Subscript Value of $\beta$ & $\beta$ & \multicolumn{2}{l}{ Confidence Interval of $\beta$} & \\
\hline \hline 0 & -6.0001 & -22.3165 & -10.3163 & & \\
1 & 0.4719 & 0.3748 & --0.5689 & \\
2 & -0.1259 & -0.3081 & --0.0563 & \\
3 & 15.5195 & 8.2927 & --22.7463 & & \\
4 & 0.0209 & -0.2791 & --0.3210 & & \\
5 & 0.0254 & -0.0490 & --0.0997 & & $S^{2}$ \\
Parameter & $\mathrm{R}^{2}$ & $\mathrm{~F}$ & $\mathrm{P}$ & 159.4828 \\
Value & 0.9726 & 213.1029 & 1.7303 & \\
\hline
\end{tabular}

From the table we can see that the $\mathrm{R}^{2}=0.9726$ shows $97.26 \%$ of variable $\mathrm{Y}$ (standard of PM2.5 levels) can be determined by the established model. The value of $\mathrm{F}$ exceeds the critical value of the $F$ test and $P$ is much smaller than that of $\alpha=0.05$, so the model from the overall look is available. So we can get a rough linear model:

$$
y=-6.0001+0.4719 x_{1}-0.1259 x_{2}+15.5195 x_{3}+0.0209 x_{4}+0.0254 x_{5}
$$

The confidence interval of Beta 0 , beta 2 , beta 4 , beta 5 contains zero, which means the impact of $\mathrm{X} 2, \mathrm{X} 4, \mathrm{X} 5$, on variable $\mathrm{Y}$ is not significant, so we focus on the consideration of $\mathrm{X} 1$ and $\mathrm{X} 3$. From the estimation of parameter values we can find that PM2.5 was affected mostly by X3 , farther more, they have a positive correlation, indicating that carbon dioxide produced by burning coal is the main internal effects of PM2.5 which means the more coal burned, the bigger the PM2.5 value is. This also explains why PM2.5 is so serious in the heating season. X1 means the content of PM10, which is positively correlated with the content of PM2.5.

Both of X1 and X2 are consistent with the actual situation, which shows that the linear fitting equation is significant. 


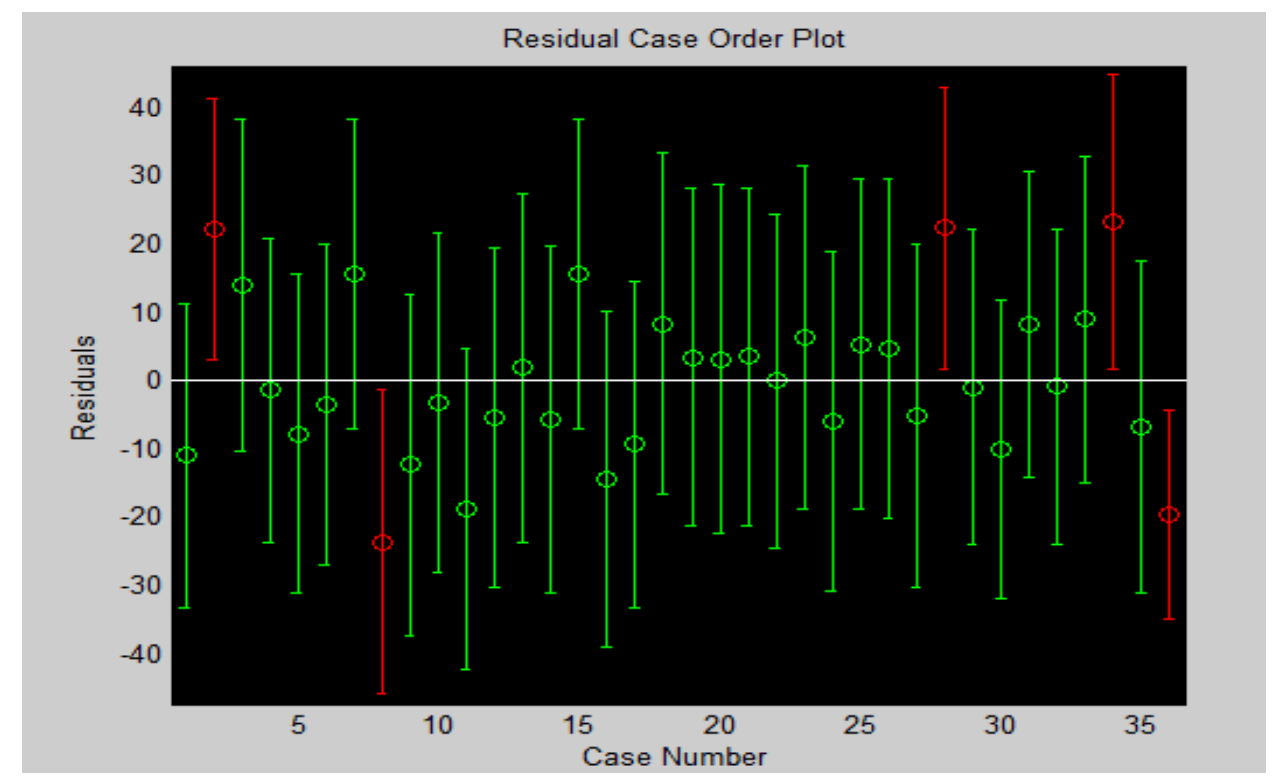

Figure 1. Residual Plot of Model One

From residual plot, we can see the distance between data residuals and zero. When the residual confidence intervals include zero, indicating that the regression model can fit the original data well, or could be regarded as outliers. From the figure, we can see only five outliers, means the regression model is fit to the actual situation.

\subsection{Model Two}

Model two is also used to analyze the factors affecting PM2.5. Model two is considered from the external causes. We use principal component analysis to build our model.

We collect related data from professional web [5]. We collected meteorological data of Baoding in 2015. The data shows as follows:

Table 4. Meteorological Data

\begin{tabular}{cccccc}
\hline Month & Temperature Dew Point & Pressure & $\begin{array}{c}\text { Wind } \\
\text { Speed }\end{array}$ & Rainfall \\
\hline 1 & -1 & -12 & 1029 & 7 & 0.8 \\
2 & 2 & -10 & 1025 & 7 & 4.3 \\
3 & 9 & -5 & 1021 & 8 & 10.2 \\
4 & 15 & 6 & 1015 & 9 & 32.2 \\
5 & 20 & 11 & 1008 & 9 & 42.9 \\
6 & 25 & 15 & 1004 & 9 & 11.8 \\
7 & 27 & 20 & 1005 & 8 & 194.5 \\
8 & 26 & 21 & 1008 & 6 & 29.7 \\
9 & 21 & 16 & 1015 & 7 & 64 \\
10 & 15 & 7 & 1020 & 7 & 11.1 \\
11 & 4 & 1 & 1027 & 6 & 35.5 \\
12 & 0 & -5 & 1029 & 6 & 0.7 \\
\hline
\end{tabular}

We used MATLAB, calculated the characteristics of numerical matrix of the five factors of twelve months' values were $0.0121,0.0372,0.5461,0.8981,3.5065$, so we can calculate the contribution rate was $0.24 \%, 0.74 \%, 10.92 \%, 17.96 \%, 70.13 \%$. 
Table 5. The Result of Influence Degree Determination

\begin{tabular}{lccc}
\hline Factors & CV & CR (\%) & CCR (\%) \\
\hline \hline Rainfall & 3.5065 & 70.13 & 70.13 \\
Wind Speed & 0.8981 & 17.96 & 88.09 \\
Pressure & 0.5461 & 10.92 & 99.01 \\
Dew point & 0.0372 & 0.74 & 99.76 \\
Temperature & 0.0121 & 0.24 & 100 \\
\hline
\end{tabular}

These data are intuitive to show that rainfall, wind speed and pressure decided effect is big in these five factors affecting PM2.5. In addition, the rainfall is bigger relative to the role of wind speed and pressure, their contribution rate and 99.01\%. Thus, we can think that these three factors are the main factors influencing PM2.5. The principal component analysis was significant.

\subsection{Model Three}

We need to look at the relationship between PM2.5 and haze. Whether PM2.5 is the main factor which influence air quality (we use AQI to measure the main factors). The data we collected is shown in Table 2 as above.

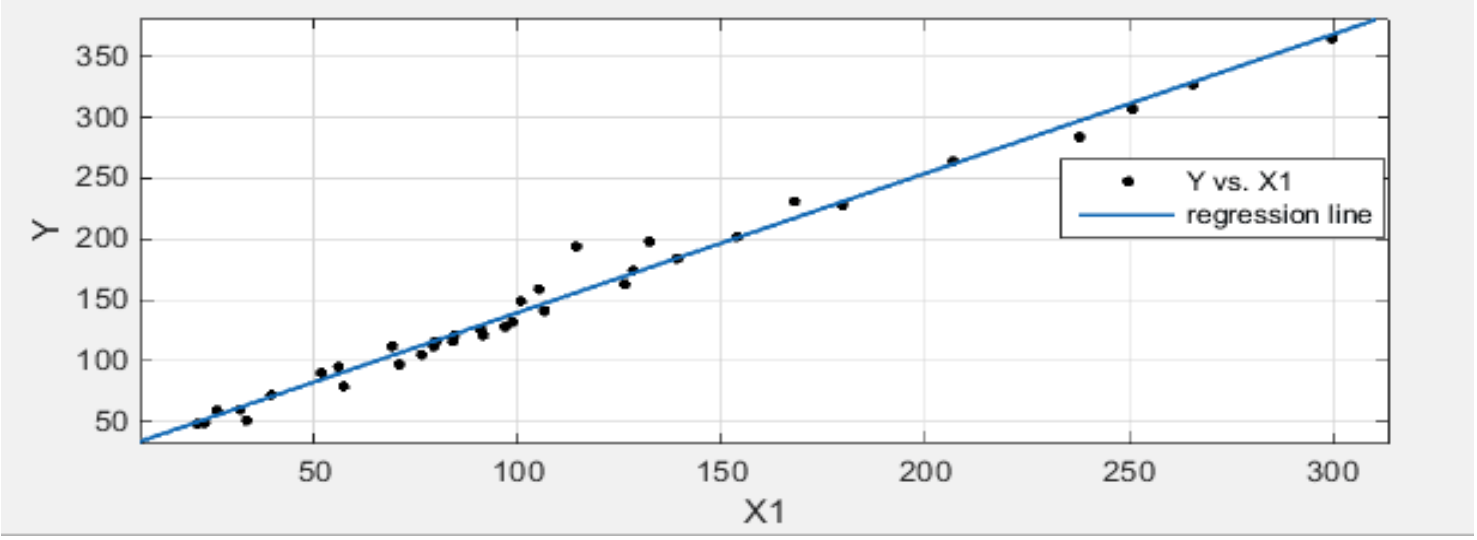

Figure2. Sample Regression Line

From the point of scatter diagram, it can be seen that PM2.5 and AQI are closely related, and there is a linear positive correlation between them. Based on this, we construct linear regression model to analyze it.

Then we establish a linear regression model.

$$
y=\beta_{0}+\beta_{1} x_{1}+\varepsilon
$$

By solving the equation with MATLAB, the regression equation of the model is

$$
y=25.1108+1.1445 x_{1}
$$

Table 6. Results of Linear Regression Model

\begin{tabular}{ccccc}
\hline Parameter & $\mathrm{R}^{2}$ & $\mathrm{~F}$ & $\mathrm{P}$ & $S^{2}$ \\
\hline Value & 0.9850 & 2229.6191 & 1.3791 & 102.6446 \\
\hline
\end{tabular}

Assuming a given $\alpha=0.05$, through the calculation and look-up table we can obtain a result that $\mathrm{F}>>\mathrm{F} 0.05(1,34)$, so there is a very significant linear relationship between PM2.5 and AQI.

In order to further verify the above regression model's fit, we use related data for residual analysis. We can see that there are only two outliers, which means that the regression model is in line with the actual situation. That is a good model fit, PM2.5 is indeed the main factors affecting the haze. PM2.5 caused by the fog and haze, while the fog and haze reflects the high value of PM2.5.

\section{Conclusion}

From the above analysis, we can see that the PM2.5 of Baoding is mainly influenced by PM10. Coal combustion, vehicle exhaust, construction site dust and is the main source of PM2.5. Time 
changes from the point of view, the pollutants in the city has obvious time variation characteristics and relative higher during heating and no heating period is relatively low. From regional distribution point of view, due to the business district is located in the downtown area, the environment is more complex, in addition the business district is located in the old city, central heating rate and clean energy utilization rate is relatively low, and the concentration of pollutants is relatively high.

Air polluted in Baoding is mainly coal-burning pollution. The particulate matter is the primary air pollutants in urban areas. Coal-fired flue gas and automobile exhaust gas and the dust from the ground is the main source of PM2.5 in Baoding. Downtown by the heat and power plant centralized heating and gas heating ratio accounted for only $30 \%$. The rest mainly is scattered coal-fired boiler. Automobile exhaust pollution is becoming more and more serious. So it is still difficult to fundamentally improve the air quality in Baoding.

\section{Reference}

[1] Shaorong Feng. factors haze influence and measures based on statistical analysis methods [M]

[2] Qingchun Li, Weihua Cao. The Characteristics of the Haze and The Analysis of Influencing Factors in Baoding. China Meteorological Administration[J]. Baoding Institute of Urban Meteorology, August 28, 2013

[3] Feng Liu, Li Yin, Xing Zhang. Part of the Linear Model in the Practice and Understanding of the Air Quality Index of Fine Particulate Matter PM 2.5 analysis. [J] . Applied Mathematics44, 2014 (9):130 - 134

[4] PM2.5 China [N].

[5] Weather Underground [N].

[6] Baidu Encyclopedia [N]. 